\title{
Spin-free relativistic no-pair ab initio core model potentials and valence basis sets for the transition metal elements $\mathrm{Sc}$ to $\mathrm{Hg}$. Part I
}

\author{
Frank Rakowitz and Christel M. Marian ${ }^{\text {a) }}$ \\ Institut für Physikalische und Theoretische Chemie, Universität Bonn, Wegelerstr. 12, D-53115 Bonn, \\ Germany \\ Luis Seijo \\ Departamento de Química, C-XIV, Universidad Autónoma de Madrid, E-28049 Madrid, Spain \\ Ulf Wahlgren \\ Fysikum, Stockholms Universitet, Box 6730, S-11385 Stockholm, Sweden
}

(Received 2 April 1998; accepted 19 November 1998)

\begin{abstract}
Relativistic one-component ab initio core model potentials are presented for first-, second-, and third-row transition elements; corresponding valence spaces comprise the $n s,(n-1) d$, and $(n$ -1) $p$ shells. Direct relativistic effects on the valence electrons are explicitly taken into account by using one-component relativistic kinetic energy and Douglas-Kroll transformed no-pair nuclear attraction interaction operators. The Coulombic part of the atomic core-valence interaction has been fitted to the corresponding all-electron mean-field operators whereas a matrix representation has been chosen for the exchange part. While not involved in the fitting process, all-electron orbital energies and radial expectation values of the valence orbitals are very well reproduced in atomic model potential calculations. Molecular test calculations have been performed on selected transition metal oxides. Employing a $[4 s, 4 p, 4 d]$ contraction of the valence basis, excellent agreement between core model potential and all-electron no-pair results is achieved for bond distances, harmonic frequencies, and dissociation energies. (C) 1999 American Institute of Physics.

[S0021-9606(99)30708-X]
\end{abstract}

\section{INTRODUCTION}

The use of one-component relativistic core potentials has become a common means to include spin-independent kinematic relativistic effects in molecular calculations. In most cases these relativistic core potentials have been generated by parameterizing nonrelativistic effective valence Hamiltonians such that they-in a least squares sense-reproduce eigenvalues or eigenvectors of a one-component relativistic atomic reference calculation. In this way relativistic effects are included solely by means of the core-valence interaction. This approximation works reasonably well because a large percentage of the relativistic effects in the valence shell is caused indirectly by a change of inner shell energies and shapes. Often, large-component Dirac-Fock ${ }^{1}$ orbitals have served as a reference to which the core potentials were fitted. Commonest in this group are the two-component relativistic effective potentials by Ermler, Ross, Christiansen, and coworkers, ${ }^{2}$ from which averaged and spin-orbit relativistic effective potentials have been extracted. Alternatively, onecomponent relativistic Hamiltonians have been used in allelectron reference calculations. The pseudopotentials by Hay and Wadt, ${ }^{3}$ Barthelat and Durand, ${ }^{4}$ the effective core potentials by the Stuttgart group, ${ }^{5}$ and the ab initio model poten-

\footnotetext{
a) Author to whom correspondence should be addressed. Present affiliation: GMD-Forschungszentrum für Informationstechnik $\mathrm{GmbH}$, Institut für Algorithmen und Wissenschaftliches Rechnen (SCAI), Schlo $\beta$ Birlinghoven, D-53754 Sankt Augustin, Germany. Electronic mail: marian@gmd.de
}

tials (AIMP) by Barandiarán and Seijo ${ }^{6,7}$ are well-known representatives of the latter derived from atomic Cowan-Griffin ${ }^{8}$ calculations.

Recently, Wittborn and Wahlgren presented relativistic AIMPs for third-row transition elements that include only the indirect relativistic effects in the core potentials. ${ }^{9}$ Direct relativistic effects on the valence electrons are treated explicitly by using the relativistic kinetic energy and electronnuclear interaction expressions of the Douglas-Kroll (DK) Hamiltonian. The latter is a spin-free relativistic Hamiltonian resulting from a transformation of a four-component no-pair Hamiltonian containing projectors to the positive energy spectrum of an electron in the (external) field of a nucleus. ${ }^{10}$

The Douglas-Kroll operator is a variationally stable one-component relativistic Hamiltonian that can be employed in all-electron treatments of molecular systems. ${ }^{11}$ This offers the possibility of testing the performance of a core model potential by direct comparison with molecular all-electron results. The same applies to the ChangPelissier-Durand operator. ${ }^{12}$ By contrast, the variational solution of the Cowan-Griffin equations is restricted to atoms. The corresponding mass-velocity and Darwin terms are unbounded from below and special boundary conditions at the nucleus are imposed on the atomic orbitals in order to handle this problem. In molecules, the all-electron Cowan-Griffin operator must be used in first-order perturbation theory in which the atomic relativistic potentials are fixed. ${ }^{8}$

We report on no-pair relativistic AIMPs and valence basis sets for the first-, second-, and third-row transition ele- 
ments. The number of primitive functions to describe the valence region is considerably smaller than in the primitive sets used by Wittborn and Wahlgren for the third-series transition elements. ${ }^{9}$ For all transition elements we compare valence orbital energies and radial expectation values from allelectron and AIMP atomic calculations to provide a quality check for the model potentials and valence basis sets. Molecular test calculations have been carried out for the electronic ground states of the group 5 and group 10 monoxides. A comparison of spectroscopic parameters obtained from either type of calculation enables us to decide whether the core-valence partition and the basis set structure have been properly chosen for molecular calculations or not.

\section{METHOD}

\section{A. All-electron spin-free Douglas-Kroll transformed no-pair Hamiltonian}

The all-electron Hamiltonian used in this work is of the Douglas-Kroll type for the one-electron terms. ${ }^{10}$ Following Samzow and Hess ${ }^{13}$ who observed that relativistic corrections to the electron-electron interaction are of minor importance in the valence shell and may thus be neglected, the electron-electron interaction is described by the plain Coulomb interaction. For a molecule with nel electrons and $N U C$ nuclei the Hamiltonian reads as

$$
H_{+}^{s f 1}=\sum_{i}^{n e l} E_{i}+\sum_{i}^{n e l} V^{s f}(i)+\sum_{i<j}^{n e l} \frac{1}{r_{i j}}+\sum_{I<J}^{N U C} \frac{Z_{I} Z_{J}}{R_{I J}} .
$$

The first term,

$$
E_{i}=\sqrt{p_{i}^{2}+m^{2}}
$$

represents the relativistic kinetic energy. The second accounts for the relativistically corrected interaction between nuclei and electrons,

$$
\begin{aligned}
V^{s f}(i)= & -A_{i}\left(V_{e x t}(i)+\vec{R}_{i} V_{e x t}(i) \vec{R}_{i}\right) A_{i} \\
& -W_{1}^{s f}(i) E_{i} W_{1}^{s f}(i)-\frac{1}{2}\left\{\left(W_{1}^{s f}(i)\right)^{2}, E_{i}\right\} .
\end{aligned}
$$

Herein, $V_{\text {ext }}(i)$ describes the (nonrelativistic) Coulomb attraction between electron $i$ and all nuclei, $E_{i}$ is the kinetic energy as defined above, and

$$
\begin{aligned}
& \vec{R}_{i}=\frac{\vec{p}_{i}}{E_{i}+m}, \\
& A_{i}=\sqrt{\frac{E_{i}+m}{2 E_{i}}},
\end{aligned}
$$

are factors resulting from the Douglas-Kroll transformation. According to a proposal by Hess, these factors are evaluated in momentum space employing the primitive molecular basis to resolve the identity. ${ }^{11}$ The same applies to $W_{1}^{s f}(i)$ which represents an integral operator with kernel

$$
W_{1}^{s f}\left(\vec{p}_{i}, \vec{p}_{i}^{\prime}\right)=A_{i}\left(\vec{R}_{i}-\vec{R}_{i}^{\prime}\right) A_{i}^{\prime} \frac{V_{e x t}\left(\vec{p}_{i}, \vec{p}_{i}^{\prime}\right)}{E_{i}+E_{i}^{\prime}} .
$$

This Hamiltonian is bounded from below ${ }^{14}$ and may thus be employed in variational procedures.

\section{B. Spin-free no-pair AIMP method}

The spin-free no-pair model potential (MP) Hamiltonian for a molecule comprising nval valence electrons and $N U C$ nuclei may be written as a sum of effective one-electron operators, the two-electron interactions in the valence space and the internuclear repulsion,

$$
H^{\mathrm{MP}}=\sum_{i=1}^{n v a l} h(i)+\sum_{i<j}^{n v a l} \frac{1}{r_{i j}}+\sum_{I<J}^{N U C} V_{I J}\left(R_{I J}\right) .
$$

The first two terms in the effective Hamiltonian for electron $i$,

$$
\begin{aligned}
h(i)= & E_{i}+V^{s f}(i)+\sum_{I}^{N U C} \frac{Z_{\text {core }}^{I}}{r_{I i}}+\sum_{I}^{N U C} V_{\text {Coul }}^{I}(i) \\
& +\sum_{I}^{N U C} V_{\text {exch }}^{I}(i)+\sum_{I}^{N U C} P^{I}(i),
\end{aligned}
$$

are identical to all-electron terms in Eq. (3). $V_{\text {Coul }}^{I}(i)$ is a radial operator representing the relativistically corrected Coulomb interaction of valence electron $i$ with the core of atom $I$,

$$
V_{\text {Coul }}^{I}(i)=V_{\text {Coul }}^{I}\left(r_{I i}\right)=-\frac{Z_{\text {core }}^{I}}{r_{I i}}+2 \sum_{c}^{\operatorname{core}(I)} J_{c}(i) .
$$

The operators $J_{c}$ are defined in a completely analogous manner to the effective Coulomb operators in Hartree-Fock theory. Here, they are calculated using the no-pair relativistic atomic core orbitals. Note that we have added a zero to the one-electron Hamiltonian [Eq. (8)] by adding and subtracting $Z_{\text {core }}^{I} / r_{I i}$. In this way, $V_{\text {Coul }}^{I}(i)$ asymptotically converges to zero much faster and can therefore more easily be approximated by a sum of Gaussians,

$$
V_{\text {Coul }}^{I}(i) \approx V_{\text {Coul }}^{I ; M P}(i)=\sum_{k} C_{I k} \frac{\exp \left(-\alpha_{I k} r_{I i}^{2}\right)}{r_{I i}} .
$$

In this work, the parameters $C_{I k}, \alpha_{I k}$ are determined through a least-squares fit to a representation of the potential $V_{C o u l}^{I}(i)$ [Eq. (9)] in the AE basis at center $I$.

Following the idea of Huzinaga et al. ${ }^{15}$ which has originally been proposed for nonrelativistic AIMPs, a nonlocal representation is employed for the exchange potential,

$$
\begin{aligned}
V_{\text {exch }}^{I}(i) & =-\sum_{c}^{\operatorname{core}(I)} K_{c}(i) \approx V_{\text {exch }}^{I, M P}(i) \\
& =\sum_{l} \sum_{m=-l}^{l} \sum_{a, b}|a l m ; I\rangle S^{-1} K S^{-1}\langle\text { blm } ; I| .
\end{aligned}
$$

The set of functions $|a l m ; I\rangle$ constitutes the intermediate basis at center $I$; each function is chosen to be a product of a radial primitive Gaussians and a spherical harmonic. $S$ is the overlap matrix and $K$ the matrix of $V_{\text {exch }}$ in this intermediate basis. In the present case we have chosen the set $|a l m ; I\rangle$ to consist of all valence primitives at least. With this choice, 
atomic calculations give identical results, regardless of whether $V_{\text {exch }}$ or $V_{\text {exch }}^{M P}$ are employed. Finally, the last term in Eq. (8) is a level shifter,

$$
P^{I}(i)=-\sum_{c}^{\operatorname{core}(I)} 2 \epsilon_{c}\left|\phi_{c}\right\rangle\left\langle\phi_{c}\right|
$$

with the core orbitals $\left(\phi_{c}\right)$ expanded in the all-electron basis. The $P^{I}$ operators shift the core orbitals to positive energies $\left(\epsilon_{c}\right)$, that is to the virtual space, and give a positive contribution to a valence orbital energy as long as the corresponding orbital is not fully orthogonal to the core; in this way, the resulting valence orbitals have an overlap with the core orbitals as small as the basis set allows.

Like in all core potential methods, the internuclear repulsion is reduced to the interaction of two effective charges:

$$
V_{I J}\left(R_{I J}\right)=\frac{\left(Z^{I}-Z_{\text {core }}^{I}\right)\left(Z^{J}-Z_{\text {core }}^{J}\right)}{R_{I J}} .
$$

Atomic no-pair relativistic all-electron calculations were performed using the program AT35 ${ }^{16}$. For the all-electron molecular calculations modified versions of either the MOLECULE-SWEDEN ${ }^{17}$ or the MOLCAS ${ }^{18}$ packages have been employed. AIMP integrals were computed using the ECPAIMP $^{19}$ code.

\section{MODEL POTENTIALS, BASIS SETS, AND ATOMIC RESULTS}

In this section we present model potentials and valence basis sets for the transition elements $\mathrm{Sc}-\mathrm{Hg}$. Further, we compare properties of valence orbitals obtained from AIMP and all-electron calculations on the atomic systems.

For the transition elements all-electron basis sets by Fægri $^{20,21}$ have been employed. The nonrelativistically optimized $16 s 11 p 8 d$ Gaussian type functions (GTFs) for the first-row transition metals (TMs) and the 20s $14 p 11 d$ sets for the second-row elements have been used without changes; the relativistic shrinkage or expansion of the orbitals is taken into account through the contraction coefficients. Each basis set has been augmented by a diffuse $d$ function required for a proper description of $s^{1} d^{x+1}$ and $s^{0} d^{x+2}$ configurations. Exponents for the first- and second-row elements were taken from the work of $\mathrm{Hay}^{22}$ and Walch et al. ${ }^{23}$ respectively. The third-row TM $22 s 16 p 13 d 8 f$ sets, on the other hand, were modified; for these elements the changes in orbital shapes, in particular, those of the valence orbitals, due to relativistic effects require an adjustment of the exponents: The two outermost $s$ exponents were scaled by a factor of 1.4 and the subsequent two-describing the $5 s$ node-by 1.25 . Furthermore, two diffuse $p$, one $d$, and one $f$ primitive were added according to the scheme described in detail by Wittborn and Wahlgren ${ }^{9}$ giving rise to a total of $22 s 18 p 14 d 9 f$ primitive GTFs.

The orbitals obtained from relativistic atomic no-pair Hartree-Fock calculations serve as a reference for the construction of the Coulomb and exchange operators. In the AIMP calculations only the outermost $n s,(n-1) p$, and ( $n$ $-1) d$ shells are treated explicitly. For all transition elements, with the exception of Pd, ground state wavefunctions have been generated. In the case of Pd the first excited $s^{1} d^{9}$ state has been chosen because the $d^{10}$ ground state does not provide meaningful contraction coefficients for the outermost $s$ shell. The parameters of the local Coulomb potentials for the two kinds of model potentials are presented in Tables $\mathrm{X}$-XII. ${ }^{24}$ The exponents of the valence basis sets have been taken from earlier work, relativistically optimized in Cowan-Griffin Hartree-Fock calculations. ${ }^{6,7,25}$ The valence basis sets of the transition elements were augmented by the same diffuse $d$ functions as were the all-electron bases. Using these primitives, we have determined contraction coefficients in atomic relativistic no-pair CASSCF calculations with $x+2$ active electrons in the active $n s$ and $(n-1) d$ shells where $x$ denotes the $d$ shell occupation in an atomic configuration with a closed valence $s$ shell. Exponents and contraction coefficients of the valence basis sets are shown in Tables XIII-XV. ${ }^{24}$ The nonlocal spectral representation of the exchange operator is actually computed during the input processing step of the molecular calculations. Since the exchange operator is represented in the complete set of valence primitives a tabulation of the matrix elements of the exchange model potential $V_{\text {exch }}^{M P}$ is not necessary. Core orbital energies $\epsilon_{c}$ and generalized contracted core orbitals required for the construction of the level shifting operators and for the calculation of the coefficients in the nonlocal representation of the exchange potential [Eq. (11)] are given in Tables XVI-XVIII. ${ }^{24}$

As a first check of the quality of the model potentials, we have computed properties of the atomic wavefunctions. In these cases we employed the basis sets in their completely uncontracted form. Energies $\epsilon$ and radial expectation values $\left(\left\langle r^{-1}\right\rangle,\langle r\rangle\right.$, and $\left.\left\langle r^{2}\right\rangle\right)$ of the valence orbitals obtained in AIMP-CASSCF calculations are compared with all-electron Hartree-Fock results in Tables I-VI. Note that neither the orbital energies nor the orbital shapes directly enter the fitting process. For most of the transition elements very good agreement is observed. At first glance, the results seem to be less satisfactory for some of the early elements. They turned out to be genuine multi-configuration cases as indicated in Tables I-VI by the weights of their main configurations. For test purposes we also carried out AIMP-HF calculations in these cases. The results show that the observed deviations between AIMP-CASSCF and AE-HF are not caused by an improper AIMP but are due to the different treatment of the valence shell. For the complete first-, second-, and third-row transition series $n s$ and $(n-1) d$ orbital energies from AIMP-HF and AE-HF calculations differ by less than $0.004 E_{H}$ or $0.01 \mathrm{eV}$. Radial expectation values are of comparable quality. Particularly noteworthy is the good agreement of the $1 / r$ expectation values since they are dominated by contributions from the inner region of the radial coordinate.

\section{MOLECULAR CALCULATIONS}

Molecular test calculations have been performed for the oxides of groups 5 and 10. The oxygen basis comprise $(10 s 5 p 2 d)$ primitive functions contracted to $[4 s 3 p 2 d]$ according to a Raffenetti scheme. ${ }^{26,27}$ Each of the TM basis 
TABLE I. Valence orbital energies $\left[E_{H}\right]$ of the first-row transition elements.

\begin{tabular}{llrlrrr}
\hline \hline & Wavefunction & $\%^{\mathrm{a}}$ & Configuration & $-\varepsilon(3 p)$ & $-\varepsilon(4 s)$ & $-\varepsilon(3 d)$ \\
\hline $\mathrm{Sc}$ & AIMP-CAS $^{\mathrm{b}}$ & 99.0 & $4 s^{2} 3 d^{1}-{ }^{2} D$ & 1.5710 & 0.2115 & 0.3310 \\
& AIMP-HF $^{\mathrm{c}}$ & 100.0 & & 1.5706 & 0.2103 & 0.3343 \\
& AE-HF $^{\mathrm{d}}$ & 100.0 & & 1.5746 & 0.2113 & 0.3356 \\
$\mathrm{Ti}$ & AIMP-CAS & 99.9 & $4 s^{2} 3 d^{2}-{ }^{3} F$ & 1.7926 & 0.2218 & 0.4298 \\
& AIMP-HF & 100.0 & & 1.7926 & 0.2214 & 0.4305 \\
& AE-HF & 100.0 & & 1.7970 & 0.2223 & 0.4315 \\
$\mathrm{~V}$ & AIMP-CAS & 99.9 & $4 s^{2} 3 d^{3}-{ }^{4} F$ & 2.0185 & 0.2317 & 0.4981 \\
& AIMP-HF & 100.0 & & 2.0185 & 0.2315 & 0.4984 \\
& AE-HF & 100.0 & & 2.0233 & 0.2324 & 0.4992 \\
$\mathrm{Cr}$ & AIMP-CAS & 100.0 & $4 s^{1} 3 d^{5}-{ }^{7} S$ & 2.0555 & 0.2246 & 0.3638 \\
& AE-HF & 100.0 & & 2.0619 & 0.2260 & 0.3664 \\
$\mathrm{Mn}$ & AIMP-CAS & 100.0 & $4 s^{2} 3 d^{5}-{ }^{6} S$ & 2.4835 & 0.2496 & 0.6251 \\
& AE-HF & 100.0 & & 2.4892 & 0.2505 & 0.6255 \\
$\mathrm{Fe}$ & AIMP-CAS & 100.0 & $4 s^{2} 3 d^{6}-{ }^{5} D$ & 2.7498 & 0.2605 & 0.6321 \\
& AE-HF & 100.0 & & 2.7558 & 0.2614 & 0.6324 \\
$\mathrm{Co}$ & AIMP-CAS & 100.0 & $4 s^{2} 3 d^{7}-{ }^{4} F$ & 3.0180 & 0.2703 & 0.6593 \\
& AE-HF & 100.0 & & 3.0256 & 0.2713 & 0.6570 \\
$\mathrm{Ni}$ & AIMP-CAS & 100.0 & $4 s^{2} 3 d^{8}-{ }^{3} F$ & 3.2945 & 0.2798 & 0.6895 \\
& AE-HF & 100.0 & & 3.3010 & 0.2806 & 0.6893 \\
$\mathrm{Cu}$ & AIMP-CAS & 100.0 & $4 s^{1} 3 d^{10}-{ }^{2} S$ & 3.3502 & 0.2425 & 0.4758 \\
& AE-HF & 100.0 & & 3.3607 & 0.2443 & 0.4789 \\
$\mathrm{Zn}$ & AIMP-CAS & 100.0 & $4 s^{2} 3 d^{10}-{ }^{1} S$ & 3.8682 & 0.2974 & 0.7615 \\
& AE-HF & 100.0 & & 3.8757 & 0.2982 & 0.7610 \\
\hline \hline
\end{tabular}

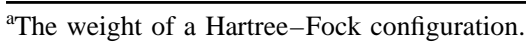

${ }^{b}$ AIMP $3 p-, 4 s-, 3 d$-valence calculation at the CASSCF level.

${ }^{c}$ AIMP $3 p-, 4 s-, 3 d$-valence calculation at the Hartree-Fock level.

${ }^{\mathrm{d}}$ All-electron calculation at the no-pair Hartree-Fock level. sets described so far was augmented by a single $p$ polarization function ${ }^{28}$ and $f$ polarization functions. The $f$ polarization function added to the first- and second-row TM bases consists of a single contraction of three GTFs. ${ }^{29}$ For the third-row transition elements the most diffuse primitive of the AE $4 f$ basis served as a polarization function. The corresponding AIMP basis sets have been augmented by a contraction of five primitive $f$ functions to maintain the orthogonality of the valence shells to the $4 f$ core $^{25}$ For a more flexible representation of the transition metal valence shells some of the most diffuse exponents in each Raffenetti contraction were added as primitives. The particular contraction scheme for the first-, second-, and third-row elements are apparent from Tables VII and VIII.

Table VII displays equilibrium bond distances and harmonic frequencies of $\mathrm{NiO}$ obtained in AIMP-CASSCF calculations with various basis sets. If the full all-electron basis is retained in the AIMP calculations, i.e., if a $[6 s / 4 p / 4 d / 1 f]$ contraction of the Fægri basis is used, only marginal deviations from the all-electron results occur. The third row in Table VII lists the values obtained from AIMP calculations in which the contractions representing the core orbitals have been deleted from the all-electron basis while the valence orbitals are left unchanged (Fægri $[3 s / 3 p / 4 d / 1 f]$ ). In this case the results deteriorate markedly. The potential energy curve is too repulsive at short bond distances and too flat at large internuclear separations. The same is true for the corresponding [911/511/6111/3] contraction of the valence basis by Casarrubios. The origin of these problems is clear cut: Since the Coulomb and exchange potentials and the level

TABLE II. Radial expectation values $\left[a_{0}\right]$ of the first-row transition elements.

\begin{tabular}{|c|c|c|c|c|c|c|c|c|c|c|c|c|}
\hline & \multirow[b]{2}{*}{ Wavefunction } & \multirow[b]{2}{*}{$\%^{\mathrm{a}}$} & \multirow[b]{2}{*}{ Configuration } & \multicolumn{3}{|c|}{$3 p$} & \multicolumn{3}{|c|}{$4 s$} & \multicolumn{3}{|c|}{$3 d$} \\
\hline & & & & $\langle 1 / r\rangle$ & $\langle r\rangle$ & $\left\langle r^{2}\right\rangle$ & $\langle 1 / r\rangle$ & $\langle r\rangle$ & $\left\langle r^{2}\right\rangle$ & $\langle 1 / r\rangle$ & $\langle r\rangle$ & $\left\langle r^{2}\right\rangle$ \\
\hline \multirow[t]{3}{*}{$\mathrm{Sc}$} & AIMP-CAS $^{b}$ & 99.0 & $4 s^{2} 3 d^{1}-{ }^{2} D$ & 1.153 & 1.171 & 1.603 & 0.321 & 3.938 & 17.857 & 0.783 & 1.720 & 3.841 \\
\hline & AIMP-HF ${ }^{c}$ & 100.0 & & 1.153 & 1.171 & 1.604 & 0.320 & 3.951 & 17.989 & 0.792 & 1.695 & 3.719 \\
\hline & $A E-H F^{d}$ & 100.0 & & 1.155 & 1.172 & 1.615 & 0.323 & 3.937 & 17.850 & 0.793 & 1.691 & 3.701 \\
\hline \multirow[t]{3}{*}{$\mathrm{Ti}$} & AIMP-CAS & 99.9 & $4 s^{2} 3 d^{2}-{ }^{3} F$ & 1.241 & 1.090 & 1.390 & 0.337 & 3.762 & 16.344 & 0.899 & 1.476 & 2.803 \\
\hline & AIMP-HF & 100.0 & & 1.241 & 1.090 & 1.390 & 0.337 & 3.766 & 16.384 & 0.901 & 1.473 & 2.790 \\
\hline & AE-HF & 100.0 & & 1.244 & 1.091 & 1.400 & 0.339 & 3.753 & 16.260 & 0.902 & 1.470 & 2.780 \\
\hline \multirow[t]{3}{*}{ V } & AIMP-CAS & 99.9 & $4 s^{2} 3 d^{3}-{ }^{4} F$ & 1.328 & 1.021 & 1.221 & 0.352 & 3.608 & 15.069 & 0.991 & 1.335 & 2.293 \\
\hline & AIMP-HF & 100.0 & & 1.328 & 1.021 & 1.221 & 0.352 & 3.610 & 15.089 & 0.992 & 1.334 & 2.288 \\
\hline & AE-HF & 100.0 & & 1.331 & 1.022 & 1.229 & 0.354 & 3.597 & 14.974 & 0.993 & 1.332 & 2.279 \\
\hline \multirow[t]{2}{*}{$\mathrm{Cr}$} & AIMP-CAS & 100.0 & $4 s^{1} 3 d^{5}-{ }^{7} S$ & 1.399 & 0.972 & 1.111 & 0.349 & 3.639 & 15.406 & 0.997 & 1.378 & 2.531 \\
\hline & AE-HF & 100.0 & & 1.402 & 0.974 & 1.121 & 0.352 & 3.622 & 15.249 & 0.998 & 1.378 & 2.541 \\
\hline \multirow[t]{2}{*}{$\mathrm{Mn}$} & AIMP-CAS & 100.0 & $4 s^{2} 3 d^{5}-{ }^{6} S$ & 1.497 & 0.909 & 0.969 & 0.379 & 3.358 & 13.117 & 1.160 & 1.139 & 1.668 \\
\hline & AE-HF & 100.0 & & 1.500 & 0.910 & 0.976 & 0.381 & 3.347 & 13.020 & 1.162 & 1.137 & 1.661 \\
\hline \multirow[t]{2}{*}{$\mathrm{Fe}$} & AIMP-CAS & 100.0 & $4 s^{2} 3 d^{6}-{ }^{5} D$ & 1.583 & 0.861 & 0.870 & 0.394 & 3.231 & 12.163 & 1.230 & 1.081 & 1.515 \\
\hline & AE-HF & 100.0 & & 1.587 & 0.862 & 0.876 & 0.397 & 3.220 & 12.076 & 1.232 & 1.079 & 1.509 \\
\hline \multirow[t]{2}{*}{ Co } & AIMP-CAS & 100.0 & $4 s^{2} 3 d^{7}-{ }^{4} F$ & 1.668 & 0.818 & 0.786 & 0.409 & 3.121 & 11.378 & 1.303 & 1.024 & 1.366 \\
\hline & AE-HF & 100.0 & & 1.671 & 0.820 & 0.792 & 0.411 & 3.111 & 11.292 & 1.304 & 1.023 & 1.362 \\
\hline \multirow[t]{2}{*}{$\mathrm{Ni}$} & AIMP-CAS & 100.0 & $4 s^{2} 3 d^{8}-{ }^{3} F$ & 1.752 & 0.780 & 0.715 & 0.423 & 3.023 & 10.694 & 1.376 & 0.972 & 1.236 \\
\hline & AE-HF & 100.0 & & 1.756 & 0.781 & 0.720 & 0.425 & 3.014 & 10.622 & 1.378 & 0.970 & 1.231 \\
\hline \multirow[t]{2}{*}{$\mathrm{Cu}$} & AIMP-CAS & 100.0 & $4 s^{1} 3 d^{10}-{ }^{2} S$ & 1.824 & 0.750 & 0.661 & 0.391 & 3.267 & 12.534 & 1.383 & 0.998 & 1.352 \\
\hline & AE-HF & 100.0 & & 1.827 & 0.753 & 0.670 & 0.391 & 3.260 & 12.505 & 1.386 & 1.000 & 1.350 \\
\hline \multirow[t]{2}{*}{$\mathrm{Zn}$} & AIMP-CAS & 100.0 & $4 s^{2} 3 d^{10}-{ }^{1} S$ & 1.918 & 0.714 & 0.599 & 0.448 & 2.854 & 9.569 & 1.523 & 0.881 & 1.022 \\
\hline & AE-HF & 100.0 & & 1.923 & 0.715 & 0.604 & 0.451 & 2.846 & 9.506 & 1.525 & 0.880 & 1.018 \\
\hline
\end{tabular}

a The weight of the Hartree-Fock configuration.

${ }^{b}$ AIMP $3 p-, 4 s-, 3 d$-valence calculation at the CASSCF level.

${ }^{c}$ AIMP $3 p-, 4 s-, 3 d$-valence calculation at the Hartree-Fock level.

${ }^{\mathrm{d}}$ The all-electron calculation at the no-pair Hartree-Fock level. 
TABLE III. Valence orbital energies $\left[E_{H}\right]$ of the second-row transition elements.

\begin{tabular}{|c|c|c|c|c|c|c|}
\hline & Wavefunction & $\%^{\mathrm{a}}$ & Configuration & $-\varepsilon(4 p)$ & $-\varepsilon(5 s)$ & $-\varepsilon(4 d)$ \\
\hline \multirow[t]{3}{*}{$\mathrm{Y}$} & AIMP-CAS ${ }^{b}$ & 92.0 & $s^{2} d^{1}-{ }^{2} D$ & 1.2980 & 0.2086 & 0.2239 \\
\hline & AIMP-HF & 100.0 & & 1.3033 & 0.2005 & 0.2302 \\
\hline & $\mathrm{AE}-\mathrm{HF}^{\mathrm{d}}$ & 100.0 & & 1.3045 & 0.2012 & 0.2314 \\
\hline \multirow[t]{3}{*}{$\mathrm{Zr}$} & AIMP-CAS & 97.5 & $s^{2} d^{2}-{ }^{3} F$ & 1.4930 & 0.2165 & 0.3109 \\
\hline & AIMP-HF & 100.0 & & 1.4954 & 0.2130 & 0.3160 \\
\hline & AE-HF & 100.0 & & 1.4954 & 0.2136 & 0.3161 \\
\hline \multirow[t]{2}{*}{$\mathrm{Nb}$} & AIMP-CAS & 100.0 & $s^{1} d^{4}-{ }^{6} D$ & 1.5782 & 0.2284 & 0.2870 \\
\hline & AE-HF & 100.0 & & 1.5784 & 0.2287 & 0.2871 \\
\hline \multirow[t]{2}{*}{ Mo } & AIMP-CAS & 100.0 & $s^{1} d^{5}-{ }^{7} S$ & 1.7501 & 0.2373 & 0.3422 \\
\hline & AE-HF & 100.0 & & 1.7506 & 0.2377 & 0.3423 \\
\hline \multirow[t]{2}{*}{$\mathrm{Tc}$} & AIMP-CAS & 100.0 & $s^{2} d^{5}-{ }^{6} S$ & 2.0631 & 0.2399 & 0.5152 \\
\hline & AE-HF & 100.0 & & 2.0645 & 0.2407 & 0.5155 \\
\hline \multirow[t]{2}{*}{$\mathrm{Ru}$} & AIMP-CAS & 100.0 & $s^{1} d^{7}-{ }^{5} F$ & 2.1398 & 0.2374 & 0.3929 \\
\hline & AE-HF & 100.0 & & 2.1414 & 0.2383 & 0.3932 \\
\hline \multirow[t]{2}{*}{$\mathrm{Rh}$} & AIMP-CAS & 100.0 & $s^{1} d^{8}-{ }^{4} F$ & 2.3374 & 0.2370 & 0.4284 \\
\hline & AE-HF & 100.0 & & 2.3392 & 0.2379 & 0.4284 \\
\hline \multirow[t]{2}{*}{$\mathrm{Pd}$} & AIMP-CAS & 100.0 & $s^{1} d^{9}-{ }^{3} D$ & 2.5382 & 0.2365 & 0.4660 \\
\hline & AE-HF & 100.0 & & 2.5404 & 0.2375 & 0.4658 \\
\hline \multirow[t]{2}{*}{$\mathrm{Ag}$} & AIMP-CAS & 100.0 & $s^{1} d^{10}-{ }^{2} S$ & 2.7409 & 0.2358 & 0.5113 \\
\hline & AE-HF & 100.0 & & 2.7428 & 0.2367 & 0.5106 \\
\hline \multirow[t]{2}{*}{$\mathrm{Cd}$} & AIMP-CAS & 100.0 & $s^{2} d^{10}-{ }^{1} S$ & 3.1153 & 0.2800 & 0.7217 \\
\hline & AE-HF & 100.0 & & 3.1180 & 0.2808 & 0.7211 \\
\hline
\end{tabular}

a The weight of the Hartree-Fock configuration.

${ }^{\mathrm{b}}$ AIMP $4 p-, 5 s-, 4 d$-valence calculation at the CASSCF level.

'AIMP $4 p-, 5 s-, 4 d$-valence calculation at the Hartree-Fock level.

${ }^{\mathrm{d}}$ The all-electron calculation at the no-pair Hartree-Fock level.

shifting operators are identical for different contractions of a given primitive basis, the errors must arise from an insufficient ability of the strongly contracted bases to keep the valence orbitals orthogonal to the core. The largest effect comes from the nonorthogonality w.r.t. the Ni $2 p$ core: Since the $2 p$ lobe of the Ni $3 p$ orbital is represented by the three innermost GTFs, a considerable improvement is observed (line 5 in Table VII) by just altering the contraction scheme

TABLE IV. Radial expectation values $\left[a_{0}\right]$ of the second-row transition elements.

\begin{tabular}{|c|c|c|c|c|c|c|c|c|c|c|c|c|}
\hline & \multirow[b]{2}{*}{ Wavefunction } & \multirow[b]{2}{*}{$\%^{\mathrm{a}}$} & \multirow[b]{2}{*}{ Configuration } & \multicolumn{3}{|c|}{$4 p$} & \multicolumn{3}{|c|}{$5 s$} & \multicolumn{3}{|c|}{$4 d$} \\
\hline & & & & $\langle 1 / r\rangle$ & $\langle r\rangle$ & $\left\langle r^{2}\right\rangle$ & $\langle 1 / r\rangle$ & $\langle r\rangle$ & $\left\langle r^{2}\right\rangle$ & $\langle 1 / r\rangle$ & $\langle r\rangle$ & $\left\langle r^{2}\right\rangle$ \\
\hline \multirow[t]{3}{*}{$\mathrm{Y}$} & AIMP-CAS $^{\mathrm{b}}$ & 92.0 & $s^{2} d^{1}-{ }^{2} D$ & 0.899 & 1.461 & 2.443 & 0.302 & 4.145 & 19.602 & 0.499 & 2.682 & 8.887 \\
\hline & AIMP-HF ${ }^{c}$ & 100.0 & & 0.898 & 1.461 & 2.443 & 0.296 & 4.223 & 20.389 & 0.529 & 2.523 & 7.832 \\
\hline & $A E-H F^{d}$ & 100.0 & & 0.901 & 1.462 & 2.451 & 0.298 & 4.210 & 20.247 & 0.531 & 2.513 & 7.773 \\
\hline \multirow[t]{3}{*}{$\mathrm{Zr}$} & AIMP-CAS & 97.5 & $s^{2} d^{2}-{ }^{3} F$ & 0.958 & 1.376 & 2.169 & 0.316 & 3.966 & 17.939 & 0.607 & 2.185 & 5.816 \\
\hline & AIMP-HF & 100.0 & & 0.958 & 1.377 & 2.169 & 0.313 & 4.000 & 18.268 & 0.614 & 2.156 & 5.647 \\
\hline & AE-HF & 100.0 & & 0.961 & 1.376 & 2.168 & 0.315 & 3.993 & 18.249 & 0.616 & 2.152 & 5.625 \\
\hline \multirow[t]{2}{*}{$\mathrm{Nb}$} & AIMP-CAS & 100.0 & $s^{1} d^{4}-{ }^{6} D$ & 1.009 & 1.312 & 1.971 & 0.323 & 3.852 & 16.978 & 0.635 & 2.117 & 5.546 \\
\hline & AE-HF & 100.0 & & 1.011 & 1.313 & 1.976 & 0.328 & 3.841 & 16.915 & 0.637 & 2.112 & 5.515 \\
\hline \multirow[t]{2}{*}{ Mo } & AIMP-CAS & 100.0 & $s^{1} d^{5}-{ }^{7} S$ & 1.064 & 1.248 & 1.783 & 0.337 & 3.698 & 15.685 & 0.701 & 1.911 & 4.487 \\
\hline & AE-HF & 100.0 & & 1.067 & 1.248 & 1.788 & 0.343 & 3.684 & 15.573 & 0.703 & 1.907 & 4.467 \\
\hline \multirow[t]{2}{*}{$\mathrm{Tc}$} & AIMP-CAS & 100.0 & $s^{2} d^{5}-{ }^{6} S$ & 1.126 & 1.182 & 1.595 & 0.353 & 3.578 & 14.751 & 0.803 & 1.646 & 3.251 \\
\hline & AE-HF & 100.0 & & 1.129 & 1.182 & 1.598 & 0.355 & 3.566 & 14.633 & 0.805 & 1.643 & 3.241 \\
\hline \multirow[t]{2}{*}{$\mathrm{Ru}$} & AIMP-CAS & 100.0 & $s^{1} d^{7}-{ }^{5} F$ & 1.173 & 1.138 & 1.481 & 0.352 & 3.571 & 14.738 & 0.814 & 1.655 & 3.363 \\
\hline & AE-HF & 100.0 & & 1.177 & 1.138 & 1.484 & 0.356 & 3.558 & 14.626 & 0.816 & 1.653 & 3.358 \\
\hline \multirow[t]{2}{*}{$\mathrm{Rh}$} & AIMP-CAS & 100.0 & $s^{1} d^{8}-{ }^{4} F$ & 1.226 & 1.091 & 1.362 & 0.358 & 3.527 & 14.424 & 0.869 & 1.553 & 2.961 \\
\hline & AE-HF & 100.0 & & 1.230 & 1.091 & 1.364 & 0.361 & 3.513 & 14.293 & 0.870 & 1.551 & 2.956 \\
\hline \multirow[t]{2}{*}{$\mathrm{Pd}$} & AIMP-CAS & 100.0 & $s^{1} d^{9}-{ }^{3} D$ & 1.279 & 1.048 & 1.258 & 0.362 & 3.491 & 14.167 & 0.922 & 1.465 & 2.632 \\
\hline & AE-HF & 100.0 & & 1.283 & 1.048 & 1.260 & 0.365 & 3.473 & 14.004 & 0.923 & 1.464 & 2.628 \\
\hline \multirow[t]{2}{*}{$\mathrm{Ag}$} & AIMP-CAS & 100.0 & $s^{1} d^{10}-{ }^{2} S$ & 1.331 & 1.010 & 1.166 & 0.365 & 3.463 & 13.984 & 0.971 & 1.387 & 2.355 \\
\hline & AE-HF & 100.0 & & 1.335 & 1.010 & 1.168 & 0.369 & 3.443 & 13.780 & 0.974 & 1.386 & 2.351 \\
\hline \multirow[t]{2}{*}{$\mathrm{Cd}$} & AIMP-CAS & 100.0 & $s^{2} d^{10}-{ }^{1} S$ & 1.391 & 0.967 & 1.069 & 0.410 & 3.099 & 11.156 & 1.054 & 1.267 & 1.936 \\
\hline & AE-HF & 100.0 & & 1.396 & 0.967 & 1.071 & 0.412 & 3.088 & 11.052 & 1.056 & 1.266 & 1.931 \\
\hline
\end{tabular}

a The weight of the Hartree-Fock configuration.

${ }^{\mathrm{b}} \mathrm{AIMP} 4 p-, 5 s-, 4 d$-valence calculation at the CASSCF level.

${ }^{c}$ AIMP $4 p-, 5 s-, 4 d$-valence calculation at the Hartree-Fock level.

${ }^{\mathrm{d}}$ All-electron calculation at the no-pair Hartree-Fock level. 
TABLE V. Valence orbital energies $\left[E_{H}\right]$ of the third-row transition elements.

\begin{tabular}{llrlrrr}
\hline \hline & Wavefunction & $\%^{\mathrm{a}}$ & \multirow{2}{*}{ Configuration } & $-\varepsilon(5 p)$ & $-\varepsilon(6 s)$ & $-\varepsilon(5 d)$ \\
\hline Hf & AIMP-CAS $^{\mathrm{b}}$ & 97.7 & $s^{2} d^{2}-{ }^{3} F$ & 1.5980 & 0.2418 & 0.2609 \\
& AIMP-HF $^{\mathrm{c}}$ & 100.0 & & 1.6007 & 0.2357 & 0.2638 \\
& AE-HF & 100.0 & & 1.6038 & 0.2365 & 0.2630 \\
Ta & AIMP-CAS & 96.0 & $s^{2} d^{3}-{ }^{4} F$ & 1.7624 & 0.2540 & 0.3166 \\
& AIMP-HF & 100.0 & & 1.7670 & 0.2485 & 0.3219 \\
& AE-HF & 100.0 & & 1.7705 & 0.2493 & 0.3207 \\
W & AIMP-CAS & 93.0 & $s^{2} d^{4}-{ }^{5} D$ & 1.9273 & 0.2664 & 0.3651 \\
& AIMP-HF & 100.0 & & 1.9351 & 0.2599 & 0.3746 \\
& AE-HF & 100.0 & & 1.9391 & 0.2608 & 0.3733 \\
Re & AIMP-CAS & 100.0 & $s^{2} d^{5}-{ }^{6} S$ & 2.1028 & 0.2694 & 0.4356 \\
& AE-HF & 100.0 & & 2.1072 & 0.2703 & 0.4342 \\
Os & AIMP-CAS & 100.0 & $s^{2} d^{6}-{ }^{5} D$ & 2.2883 & 0.2831 & 0.4532 \\
& AE-HF & 100.0 & & 2.2932 & 0.2840 & 0.4517 \\
Ir & AIMP-CAS & 100.0 & $s^{2} d^{7}-{ }^{4} F$ & 2.4725 & 0.2946 & 0.4859 \\
& AE-HF & 100.0 & & 2.4780 & 0.2957 & 0.4844 \\
Pt & AIMP-CAS & 100.0 & $s^{1} d^{9}-{ }^{3} D$ & 2.5553 & 0.2892 & 0.4147 \\
& AE-HF & 100.0 & & 2.5621 & 0.2910 & 0.4138 \\
Au & AIMP-CAS & 100.0 & $s^{1} d^{10}-{ }^{2} S$ & 2.7372 & 0.2877 & 0.4547 \\
& AE-HF & 100.0 & & 2.7445 & 0.2895 & 0.4538 \\
Hg & AIMP-CAS & 100.0 & $s^{2} d^{10}-{ }^{1} S$ & 3.0397 & 0.3240 & 0.6066 \\
& AE-HF & 100.0 & & 3.0466 & 0.3257 & 0.6043 \\
\hline \hline
\end{tabular}

${ }^{\mathrm{a}}$ The weight of the Hartree-Fock configuration.

${ }^{\mathrm{b}}$ AIMP $5 p-, 6 s-, 5 d$-valence calculation at the CASSCF level.

${ }^{c}$ AIMP $5 p-, 6 s-, 5 d$-valence calculation at the Hartree-Fock level.

${ }^{\mathrm{d}}$ All-electron calculation at the no-pair Hartree-Fock level.

of the $p$ primitives from [511] to [521] without changing the number of contracted $p$ orbitals. The decontraction of the fourth $p$ primitive rectifies most of the remaining error. If, in addition, the expansion coefficient of a further $s$ function is allowed to float, the results become nearly identical to both the results in the completely uncontracted valence basis and the all-electron results. Summarizing, we find that an AIMP calculation employing a $[4 s / 4 p / 4 d / 1 f]$ valence basis on $\mathrm{Ni}$ yields excellent agreement with all-electron results on $\mathrm{NiO}$; if, for reasons of efficiency, a smaller basis has to be used, a [ $3 s / 3 p / 4 d / 1 f]$ set with contraction scheme [911/521/6111/3] still gives reasonable agreement.

In the following, all calculations for first- and secondrow TM oxides with AIMPs corresponding to $[\mathrm{Mg}]$ and $[\mathrm{Zn}]$ cores, respectively, have been carried out using a $[4 s / 4 p / 4 d / 1 f]$ contraction of the valence basis. For the thirdrow element AIMPs with $[\mathrm{C} d, 4 f]$ core a $[4 s / 4 p / 4 d / 2 f]$ contraction has been employed. In order to make the comparison between AIMP and AE results more transparent, we have repeated the $\mathrm{AE}$ calculations with the decontracted primitives replaced by the corresponding GTFs from the AIMP valence basis.

Table VIII displays equilibrium bond distances, harmonic vibrational frequencies, and dissociation energies of the ground or low-lying excited electronic states of the group 5 oxides $\mathrm{VO}, \mathrm{NbO}$, and $\mathrm{TaO}$ and the group 10 oxides $\mathrm{NiO}$, $\mathrm{PdO}$, and PtO. Unless noted otherwise, all calculations have been performed at the Hartree-Fock level. For the group 5 oxides we have chosen a ${ }^{4} \Sigma^{-}$state with electronic configuration $\sigma_{\mathrm{O} 2 s}^{2} \sigma^{2} \pi^{4} \sigma^{\prime 1} \delta^{2}$, the electronic ground state of $\mathrm{VO}^{30}$ and $\mathrm{NbO} .{ }^{31} \mathrm{NiO}$ exhibits a ${ }^{3} \Sigma^{-}$ground state with electron configuration $\sigma_{\mathrm{O} 2 s}^{2} \sigma^{2} \pi^{4} \delta^{4} \sigma^{\prime 2} \pi^{\prime 2}{ }^{30}$ The corresponding state in PdO is not bound at the Hartree-Fock level. In this case the test calculations have been performed for the $\sigma_{\mathrm{O} 2 s}^{2} \sigma^{2} \pi^{4} \delta^{4} \sigma^{\prime 1} \pi^{\prime 3}\left({ }^{3} \Pi\right)$ state. Due to convergence prob-

TABLE VI. Radial expectation values $\left[a_{0}\right]$ of the third-row transition elements.

\begin{tabular}{|c|c|c|c|c|c|c|c|c|c|c|c|c|}
\hline & \multirow[b]{2}{*}{ Wavefunction } & \multirow[b]{2}{*}{$\%^{\mathrm{a}}$} & \multirow[b]{2}{*}{ Configuration } & \multicolumn{3}{|c|}{$5 p$} & \multicolumn{3}{|c|}{$6 s$} & \multicolumn{3}{|c|}{$5 d$} \\
\hline & & & & $\langle 1 / r\rangle$ & $\langle r\rangle$ & $\left\langle r^{2}\right\rangle$ & $\langle 1 / r\rangle$ & $\langle r\rangle$ & $\left\langle r^{2}\right\rangle$ & $\langle 1 / r\rangle$ & $\langle r\rangle$ & $\left\langle r^{2}\right\rangle$ \\
\hline \multirow[t]{3}{*}{$\mathrm{Hf}$} & AIMP-CAS & 97.7 & \multirow[t]{3}{*}{$s^{2} d^{2}-{ }^{3} F$} & 0.958 & 1.355 & 2.083 & 0.340 & 3.684 & 15.527 & 0.552 & 2.380 & 6.905 \\
\hline & AIMP-HF ${ }^{c}$ & 100.0 & & 0.958 & 1.355 & 2.084 & 0.336 & 3.725 & 15.897 & 0.561 & 2.342 & 6.675 \\
\hline & $\mathrm{AE}-\mathrm{HF}^{\mathrm{d}}$ & 100.0 & & 0.963 & 1.354 & 2.086 & 0.339 & 3.705 & 15.678 & 0.560 & 2.343 & 6.682 \\
\hline \multirow[t]{3}{*}{$\mathrm{Ta}$} & AIMP-CAS & 96.0 & \multirow[t]{3}{*}{$s^{2} d^{3}-{ }^{4} F$} & 0.996 & 1.306 & 1.935 & 0.355 & 3.533 & 14.301 & 0.610 & 2.146 & 5.554 \\
\hline & AIMP-HF & 100.0 & & 0.996 & 1.306 & 1.934 & 0.352 & 3.570 & 14.618 & 0.616 & 2.122 & 5.424 \\
\hline & AE-HF & 100.0 & & 1.001 & 1.305 & 1.936 & 0.355 & 3.549 & 14.401 & 0.616 & 2.123 & 5.428 \\
\hline \multirow[t]{3}{*}{ W } & AIMP-CAS & 93.0 & \multirow[t]{3}{*}{$s^{2} d^{4}-{ }^{5} D$} & 1.033 & 1.262 & 1.803 & 0.371 & 3.340 & 13.235 & 0.657 & 1.986 & 4.731 \\
\hline & AIMP-HF & 100.0 & & 1.033 & 1.261 & 1.803 & 0.367 & 3.437 & 13.569 & 0.664 & 1.965 & 4.619 \\
\hline & AE-HF & 100.0 & & 1.039 & 1.261 & 1.804 & 0.369 & 3.418 & 13.374 & 0.664 & 1.966 & 4.622 \\
\hline \multirow[t]{2}{*}{$\mathrm{Re}$} & AIMP-CAS & 100.0 & \multirow[t]{2}{*}{$s^{2} d^{5}-{ }^{6} S$} & 1.070 & 1.220 & 1.686 & 0.380 & 3.329 & 12.741 & 0.709 & 1.836 & 4.006 \\
\hline & AE-HF & 100.0 & & 1.076 & 1.219 & 1.686 & 0.383 & 3.309 & 12.555 & 0.709 & 1.836 & 4.009 \\
\hline \multirow[t]{2}{*}{ Os } & AIMP-CAS & 100.0 & \multirow[t]{2}{*}{$s^{2} d^{6}-{ }^{5} D$} & 1.108 & 1.181 & 1.578 & 0.395 & 3.207 & 11.833 & 0.746 & 1.752 & 3.655 \\
\hline & AE-HF & 100.0 & & 1.115 & 1.180 & 1.578 & 0.398 & 3.188 & 11.666 & 0.746 & 1.753 & 3.656 \\
\hline \multirow[t]{2}{*}{$\mathrm{Ir}$} & AIMP-CAS & 100.0 & \multirow[t]{2}{*}{$s^{2} d^{7}-{ }^{4} F$} & 1.146 & 1.145 & 1.483 & 0.409 & 3.105 & 11.106 & 0.784 & 1.671 & 3.321 \\
\hline & AE-HF & 100.0 & & 1.153 & 1.144 & 1.482 & 0.412 & 3.088 & 10.951 & 0.784 & 1.671 & 3.322 \\
\hline \multirow[t]{2}{*}{$\mathrm{Pt}$} & AIMP-CAS & 100.0 & \multirow[t]{2}{*}{$s^{1} d^{9}-{ }^{3} D$} & 1.177 & 1.116 & 1.410 & 0.407 & 3.118 & 11.231 & 0.797 & 1.662 & 3.328 \\
\hline & AE-HF & 100.0 & & 1.185 & 1.115 & 1.410 & 0.411 & 3.099 & 11.066 & 0.797 & 1.662 & 3.326 \\
\hline \multirow[t]{2}{*}{$\mathrm{Au}$} & AIMP-CAS & 100.0 & \multirow[t]{2}{*}{$s^{1} d^{10}-{ }^{2} S$} & 1.214 & 1.085 & 1.332 & 0.413 & 3.084 & 11.017 & 0.834 & 1.585 & 3.013 \\
\hline & AE-HF & 100.0 & & 1.222 & 1.084 & 1.331 & 0.416 & 3.066 & 10.851 & 0.835 & 1.584 & 3.012 \\
\hline \multirow[t]{2}{*}{$\mathrm{Hg}$} & AIMP-CAS & 100.0 & \multirow[t]{2}{*}{$s^{2} d^{10}-{ }^{1} S$} & 1.256 & 1.050 & 1.246 & 0.445 & 2.864 & 9.479 & 0.894 & 1.470 & 2.560 \\
\hline & AE-HF & 100.0 & & 1.265 & 1.049 & 1.246 & 0.451 & 2.844 & 9.324 & 0.895 & 1.470 & 2.561 \\
\hline
\end{tabular}

The weight of the Hartree-Fock configuration.

${ }^{\mathrm{b}}$ AIMP $5 p-, 6 s-, 5 d$-valence calculation at the CASSCF level.

${ }^{c}$ AIMP $5 p-, 6 s-, 5 d$-valence calculation at the Hartree-Fock level.

${ }^{\mathrm{d}}$ All-electron calculation at the no-pair Hartree-Fock level. 
TABLE VII. Equilibrium bond distance $R_{e}$, harmonic frequency $\omega_{e}$, and dissociation energy $D_{e}$ of NiO: Basis set dependence of AIMP-CASSCF values and comparison with all-electron results.

\begin{tabular}{llccc}
\hline \hline Core & \multicolumn{1}{c}{ Contracted basis set $^{\mathrm{a}}$} & $R_{e}(\AA)$ & $\omega_{e}\left(\mathrm{~cm}^{-1}\right)$ & $D_{e}(\mathrm{eV})$ \\
\hline AE & $\mathrm{F}[16,16,16,16,1,1 / 11,11,1,1 / 9,1,1,1 / 3]$ & 1.672 & 779 & $-1.66^{\mathrm{b}}$ \\
AIMP & $\mathrm{F}[16,16,16,16,1,1 / 11,11,1,1 / 9,1,1,1 / 3]$ & 1.669 & 782 & -1.64 \\
AIMP & $\mathrm{F}[16,1,1 / 11,1,1 / 9,1,1,1 / 3]$ & 1.694 & 730 & -1.84 \\
AIMP & $\mathrm{V}[9,1,1 / 5,1,1 / 6,1,1,1 / 3]$ & 1.696 & 722 & -1.81 \\
AIMP & $\mathrm{V}[9,1,1 / 5,2,1 / 6,1,1,1 / 3]$ & 1.684 & 737 & -1.75 \\
AIMP & $\mathrm{V}[9,1,1 / 5,1,1,1 / 6,1,1,1 / 3]$ & 1.678 & 759 & -1.68 \\
AIMP & $\mathrm{V}[9,1,1,1 / 5,1,1,1 / 6,1,1,1 / 3]$ & 1.673 & 769 & -1.65 \\
AIMP & $\mathrm{V}[1,1,1,1,1,1,1,1,1 / 1,1,1,1,1,1 / 1,1,1,1,1,1 / 3]$ & 1.672 & 776 & -1.63 \\
\hline \hline
\end{tabular}

${ }^{\mathrm{a}} \mathrm{F}$ denotes the $\mathrm{Ni}$ basis by Fægri $;{ }^{20} \mathrm{~V}$ the Ni valence basis by Casarrubios and Seijo. ${ }^{25}$

${ }^{\mathrm{b}}$ The negative value indicates that the ${ }^{3} \Sigma^{-}$state of $\mathrm{NiO}$ is metastable with respect to dissociation into $\mathrm{Ni}\left({ }^{3} F\right)$ and $\mathrm{O}\left({ }^{3} P\right)$ at the Hartree-Fock level.

lems of the single determinant representation of the ${ }^{3} \Sigma^{-}$ state of PtO, we have carried out small CASSCF calculations for this state with six active electrons in the $\pi$ and $\pi^{\prime}$ orbitals. Dissociation energies have been computed in a supermolecule approach i.e., as energy differences between (1) the molecular energies at equilibrium bond distance and (2) the energy of a high-spin state at the internuclear separation of $1000 a_{0}$. The use of a super-molecule approach for the determination of dissociation energies is crucial for a balanced matrix representation of the momentum-dependent terms in the no-pair Hamiltonian [Eq. (3)]: Employing different resolutions of the identity for the molecule and the separated atom limit leads to completely unreasonable results.

Regarding bond distances, vibrational frequencies, and dissociation energies, excellent agreement between AIMP and $\mathrm{AE}$ results is observed for the late transition metal oxides. Equilibrium bond distances are reproduced by the AIMP treatment with deviations of less than $0.01 \AA$, and harmonic frequencies agree to within a few $\mathrm{cm}^{-1}$ ranging from complete agreement (best case) to $20 \mathrm{~cm}^{-1}$ (worst case). Dissociation energies differ by at most $0.07 \mathrm{eV}$. Somewhat larger errors are observed for the early transition metal oxides. Interestingly, the ${ }^{4} \Sigma^{-}$states of $\mathrm{VO}$ and $\mathrm{TaO}$ which correlate with $\mathrm{V}^{4} F_{g}\left(4 s^{2} d^{3}\right)$ and $\mathrm{O}^{3} P_{g}$ in the separated atom limit are slightly overbound in the AIMP treatment; the overbinding is also apparent from the computed equilibrium bond distances which are too short. On the contrary the dissociation energy of the corresponding state in $\mathrm{NbO}$ which originates from $\mathrm{O}^{3} P_{g}$ and $\mathrm{Nb}$ in its ${ }^{6} D_{g}\left(5 s^{1} d^{4}\right)$ ground state is slightly underestimated. Several reasons may be thought of as being responsible for these deviations. The most obvious one is the neglect of the $(n-1) s$ shell polarization which is known to play an important role in compounds of the early transition metals but which is not taken into account in the AIMPs with $[\mathrm{Mg}],[\mathrm{Zn}]$, and $[\mathrm{Cd}, 4 f]$ cores, respectively. A further approximation to be checked is the completeness of the matrix representation of the exchange operator [Eq. (11)] and the relativistic kinematic factors [Eqs. (4) and (5)]. For this purpose we have constructed a set of small-core ([Ne], $[\mathrm{Ar}, 3 d]$, and $[\mathrm{Kr}, 4 d, 4 f]$, respectively) AIMPs and corresponding basis sets. Technical details will be presented in a forthcoming publication. ${ }^{32}$ Furthermore, we have designed a series of AIMP calculations with the intermediate bases $|a l m ; I\rangle$ augmented by a selection from the corresponding TM AE basis set. The latter type of basis set is denoted by the label aug.val in Table IX.

Let us focus on the $(n-1) s$ shell polarization effect first. Enlarging the valence space by including the $(n-1) s$ shell has a marked effect only on the dissociation energies of the early transition metal oxides. In all cases $D_{e}$ is decreased

TABLE VIII. Equilibrium bond distances $R_{e}$, harmonic frequencies $\omega_{e}$, and dissociation energies $D_{e}$ of low-lying electronic states of the group 5 and 10 oxides obtained at the all-electron (AE) and AIMP valence Hartree-Fock levels.

\begin{tabular}{|c|c|c|c|c|c|c|}
\hline Molecule & State & Calculation & Contracted TM basis set & $R_{e}(\AA)$ & $\omega_{e}\left(\mathrm{~cm}^{-1}\right)$ & $D_{e}(\mathrm{eV})$ \\
\hline \multirow[t]{2}{*}{ VO } & ${ }^{4} \Sigma^{-}\left(\sigma \delta^{2}\right)$ & $\mathrm{AE}$ & {$[16,16,16,16,1,1,1 / 11,11,1,1,1 / 9,1,1,1 / 3]$} & 1.555 & 1178 & 1.53 \\
\hline & & AIMP & {$[9,1,1,1 / 5,1,1,1 / 6,1,1,1 / 3]$} & 1.542 & 1199 & 1.72 \\
\hline \multirow[t]{2}{*}{$\mathrm{NbO}$} & ${ }^{4} \Sigma^{-}\left(\sigma \delta^{2}\right)$ & $\mathrm{AE}$ & {$[20,20,20,20,20,1,1,1 / 14,14,14,1,1,1 / 12,12,1,1,1 / 3]$} & 1.658 & 1105 & 3.51 \\
\hline & & AIMP & {$[11,1,1,1 / 7,1,1,1 / 7,1,1,1 / 3]$} & 1.657 & 1080 & 3.46 \\
\hline \multirow[t]{2}{*}{$\mathrm{TaO}$} & ${ }^{4} \Sigma^{-}\left(\sigma \delta^{2}\right)$ & $\mathrm{AE}$ & {$[22,22,22,22,22,22,1,1,1 / 18,18,18,18,1,1,1 / 14,14,14,1,1,1 / 9,1]$} & 1.693 & 1070 & 4.21 \\
\hline & & AIMP & {$[13,1,1,1 / 9,1,1,1 / 9,1,1,1 / 5,1]$} & 1.683 & 1068 & 4.42 \\
\hline \multirow[t]{2}{*}{$\mathrm{NiO}$} & ${ }^{3} \Sigma^{-}\left(\sigma^{2} \delta^{4} \pi^{2}\right)$ & $\mathrm{AE}$ & {$[16,16,16,16,1,1,1 / 11,11,1,1,1 / 9,1,1,1 / 3]$} & 1.674 & 773 & $-1.72^{\mathrm{a}}$ \\
\hline & & AIMP & {$[9,1,1,1 / 5,1,1,1 / 6,1,1,1 / 3]$} & 1.673 & 769 & -1.65 \\
\hline \multirow[t]{2}{*}{$\mathrm{PdO}$} & ${ }^{3} \Pi\left(\sigma^{1} \pi^{1}\right)^{\mathrm{b}}$ & $\mathrm{AE}$ & {$[20,20,20,20,20,1,1,1 / 14,14,14,1,1,1 / 12,12,1,1,1 / 3]$} & 1.981 & 549 & 0.75 \\
\hline & & AIMP & {$[11,1,1,1 / 7,1,1,1 / 7,1,1,1 / 3]$} & 1.980 & 549 & 0.80 \\
\hline \multirow[t]{2}{*}{$\mathrm{PtO}$} & ${ }^{3} \Sigma^{-}\left(\sigma^{2} \delta^{4} \pi^{2}\right)$ & $\mathrm{AE}$ & {$[22,22,22,22,22,22,1,1,1 / 18,18,18,18,1,1,1 / 14,14,14,1,1,1 / 9,1]$} & 1.808 & 686 & 0.89 \\
\hline & & AIMP & {$[13,1,1,1 / 9,1,1,1 / 9,1,1,1 / 5,1]$} & 1.799 & 706 & 0.92 \\
\hline
\end{tabular}

${ }^{a}$ The negative value indicates that the ${ }^{3} \Sigma^{-}$state of $\mathrm{NiO}$ is metastable with respect to dissociation into $\mathrm{Ni}\left({ }^{3} F\right)$ and $\mathrm{O}\left({ }^{3} P\right)$ at the Hartree-Fock level.

${ }^{\mathrm{b}} \mathrm{The} \sigma^{2} \delta^{4} \pi^{2}-{ }^{3} \Sigma^{-}$state of PdO is not bound at the HF level.

${ }^{\mathrm{c}}$ The CASSCF calculation distributing 6 electrons in the $\pi$ and $\pi^{\prime}$ orbitals; HF calculations were not convergent. 
TABLE IX. Comparison of spectroscopic parameters obtained from AE, large-core ([Mg], $[\mathrm{Zn}]$, and $[\mathrm{Cd}, 4 f]$ core, respectively) and small-core ([Ne], $[\mathrm{Ar}, 3 d]$, and $[\mathrm{Kr}, 4 d, 4 f]$ core, respectively) AIMP calculations. The labels val and aug.val denote different intermediate basis sets (see text).

\begin{tabular}{|c|c|c|c|c|c|c|c|c|}
\hline \multirow[b]{2}{*}{ Molecule } & \multirow[b]{2}{*}{ State } & & \multirow[b]{2}{*}{ Core } & \multicolumn{2}{|c|}{ Representation } & \multirow[b]{2}{*}{$R_{e}(\AA)$} & \multirow[b]{2}{*}{$\omega_{e}\left(\mathrm{~cm}^{-1}\right)$} & \multirow[b]{2}{*}{$D_{e}(\mathrm{eV})$} \\
\hline & & & & Exchange & Relativistic & & & \\
\hline \multirow[t]{5}{*}{ VO } & \multirow[t]{5}{*}{${ }^{4} \Sigma^{-}$} & AIMP & {$[\mathrm{Mg}]$} & val & val & 1.542 & 1199 & 1.72 \\
\hline & & AIMP & {$[\mathrm{Mg}]$} & aug.val & aug.val & 1.545 & 1187 & 1.63 \\
\hline & & AIMP & {$[\mathrm{Ne}]$} & val & val & 1.551 & 1191 & 1.59 \\
\hline & & AIMP & {$[\mathrm{Ne}]$} & aug.val & aug.val & 1.552 & 1185 & 1.54 \\
\hline & & $\mathrm{AE}$ & - & - & $a e$ & 1.555 & 1178 & 1.53 \\
\hline \multirow[t]{5}{*}{$\mathrm{NbO}$} & \multirow[t]{5}{*}{${ }^{4} \Sigma^{-}$} & AIMP & {$[\mathrm{Zn}]$} & val & val & 1.657 & 1080 & 3.46 \\
\hline & & AIMP & {$[\mathrm{Zn}]$} & aug.val & aug.val & 1.655 & 1099 & 3.59 \\
\hline & & AIMP & {$[\mathrm{Ar}, 3 d]$} & val & val & 1.665 & 1089 & 3.35 \\
\hline & & AIMP & {$[\mathrm{Ar}, 3 d]$} & aug.val & aug.val & 1.661 & 1110 & 3.53 \\
\hline & & $\mathrm{AE}$ & - & - & $a e$ & 1.658 & 1105 & 3.51 \\
\hline \multirow[t]{5}{*}{$\mathrm{TaO}$} & \multirow[t]{5}{*}{${ }^{4} \Sigma^{-}$} & AIMP & {$[\mathrm{Cd}, 4 f]$} & val & val & 1.683 & 1068 & 4.42 \\
\hline & & AIMP & {$[\mathrm{Cd}, 4 f]$} & aug.val & aug.val & 1.686 & 1061 & 4.33 \\
\hline & & AIMP & {$[\mathrm{Kr}, 4 d, 4 f]$} & val & val & 1.686 & 1074 & 4.36 \\
\hline & & AIMP & {$[\mathrm{Kr}, 4 d, 4 f]$} & aug.val & aug.val & 1.689 & 1066 & 4.26 \\
\hline & & $\mathrm{AE}$ & - & - & $a e$ & 1.693 & 1070 & 4.21 \\
\hline \multirow[t]{5}{*}{$\mathrm{NiO}$} & \multirow[t]{5}{*}{${ }^{3} \Sigma^{-}$} & AIMP & {$[\mathrm{Mg}]$} & val & val & 1.673 & 769 & -1.65 \\
\hline & & AIMP & {$[\mathrm{Mg}]$} & aug.val & aug.val & 1.675 & 771 & -1.68 \\
\hline & & AIMP & {$[\mathrm{Ne}]$} & val & val & 1.677 & 768 & -1.66 \\
\hline & & AIMP & {$[\mathrm{Ne}]$} & aug.val & aug.val & 1.678 & 769 & -1.70 \\
\hline & & $\mathrm{AE}$ & - & - & $a e$ & 1.674 & 773 & -1.72 \\
\hline \multirow[t]{5}{*}{$\mathrm{PdO}$} & \multirow[t]{5}{*}{${ }^{3} \Pi$} & AIMP & {$[\mathrm{Zn}]$} & val & val & 1.980 & 549 & 0.80 \\
\hline & & AIMP & {$[\mathrm{Zn}]$} & aug.val & aug.val & 1.974 & 548 & 0.81 \\
\hline & & AIMP & {$[\mathrm{Ar}, 3 d]$} & val & val & 1.987 & 547 & 0.78 \\
\hline & & AIMP & {$[\mathrm{Ar}, 3 d]$} & aug.val & aug.val & 1.977 & 548 & 0.79 \\
\hline & & $\mathrm{AE}$ & - & - & $a e^{3}$ & 1.981 & 549 & 0.75 \\
\hline \multirow[t]{5}{*}{$\mathrm{PtO}$} & \multirow[t]{5}{*}{${ }^{3} \Sigma^{-}$} & AIMP & {$[\mathrm{Cd}, 4 f]$} & val & val & 1.799 & 706 & 0.92 \\
\hline & & AIMP & {$[\mathrm{Cd}, 4 f]$} & aug.val & aug.val & 1.807 & 695 & 0.87 \\
\hline & & AIMP & {$[\mathrm{Kr}, 4 d, 4 f]$} & val & val & 1.801 & 701 & 0.91 \\
\hline & & AIMP & {$[\mathrm{Kr}, 4 d, 4 f]$} & aug.val & aug.val & 1.812 & 685 & 0.83 \\
\hline & & $\mathrm{AE}$ & - & - & $a e$ & 1.808 & 686 & 0.89 \\
\hline
\end{tabular}

bringing the values for $\mathrm{VO}$ and $\mathrm{TaO}$ into better agreement with the AE values. For $\mathrm{NbO}$, which already in the AIMP $[\mathrm{Zn}]$ calculation exhibits too small a dissociation energy the deviation is seemingly increased. The latter results point to a cancellation of errors in the $\mathrm{NbO}$ large-core calculation. The remaining errors are essentially removed by improving the matrix representation of the exchange and relativistic operators (entry aug.val in Table IX).

\section{CONCLUSIONS}

In this work, we provide relativistic ab initio no-pair model potentials and valence basis sets for the transition elements $\mathrm{Sc}-\mathrm{Hg}$. A comparison of atomic properties obtained from AIMP Hartree-Fock and all-electron HF calculations shows that $n s$ and $(n-1) d$ valence orbital energies differ by less than $0.004 E_{H}(0.01 \mathrm{eV})$ for the complete first-, second-, and third-row transition series. Radial expectation values are of comparable quality.

AIMP calculations on late TM oxides with $[\mathrm{Mg}],[\mathrm{Zn}]$, and $[\mathrm{Cd}, 4 \mathrm{f}]$ cores, respectively, and employing a $[4 s / 4 p / 4 d / 1 f]$ contraction of the TM valence basis are in excellent agreement with all-electron results; if, for reasons of efficiency, a smaller basis has to be used, a [3s/3p/4d/1f] contraction still gives reasonable agreement. The performance of the AIMP method is slightly less satisfactory for the early transition metal oxides, if the high accuracy of the results on the late TM oxides is taken as a reference. The deviations from the corresponding all-electron values fall, however, well in the range of other types of effective core potentials. It is shown that also in these cases the spectroscopic parameters can be brought into excellent agreement with the AE values, if the $(n-1) s$ shell is included in the valence space and the intermediate basis sets-used for representing the exchange and no-pair operators-are improved.

\section{ACKNOWLEDGMENTS}

Financial support by the German Research Council (DFG Priority Project "Relativistic effects," Ma 1051/2-2) and MEC, Spain, (PB95-0201) is gratefully acknowledged. Further, we express our gratitude to the European Science Foundation (REHE-Program), the Spanish and German Academic Exchange Services (Acciones Integradas, Contract No. HA1997-0097, and DAAD, Contract No. 314-AI-e-dr) for traveling funds. F.R. wishes to thank the members of the 
Theoretical Chemistry group at the "Universidad Autónoma de Madrid" for their warm hospitality during his stay in Madrid.

${ }^{1}$ J. P. Desclaux, Atomic Data Nucl. Data Tables 12, 311 (1973).

${ }^{2}$ W. C. Ermler, R. B. Ross, and P. A. Christiansen, Adv. Quantum Chem. 19, 139 (1988).

${ }^{3}$ P. J. Hay and W. R. Wadt, J. Chem. Phys. 82, 299 (1985).

${ }^{4}$ J. C. Barthelat and P. Durand, Gazz. Chim. Ital. 108, 225 (1978).

${ }^{5}$ D. Andrae, U. Häußermann, M. Dolg, and H. Preuss, Theor. Chim. Acta 77, 123 (1990).

${ }^{6}$ Z. Barandiarán and L. Seijo, J. Chem. Phys. 93, 5843 (1990).

${ }^{7}$ Z. Barandiarán and L. Seijo, Can. J. Chem. 70, 409 (1991).

${ }^{8}$ R. D. Cowan and D. C. Griffin, J. Opt. Soc. Am. 66, 1010 (1976).

${ }^{9}$ C. Wittborn and U. Wahlgren, Chem. Phys. 201, 357 (1995).

${ }^{10}$ M. Douglas and N. M. Kroll, Ann. Phys. (N.Y.) 82, 89 (1974).

${ }^{11}$ B. A. Heß, Phys. Rev. A 33, 3742 (1986).

${ }^{12}$ C. Chang, M. Pélissier, and P. Durand, Phys. Scr. 34, 394 (1986).

${ }^{13}$ R. Samzow and B. A. Heß, Chem. Phys. Lett. 184, 491 (1991).

${ }^{14}$ G. Hardekopf and J. Sucher, Phys. Rev. A 30, 703 (1984).

${ }^{15}$ S. Huzinaga, L. Seijo, Z. Barandiarán, and M. Klobukowski, J. Chem. Phys. 86, 2132 (1987).

${ }^{16}$ AT35 is an atomic SCF program originally written by B. O. Roos and modified later by L. Gianolio. Relativistic operators were introduced by B. A. Hess.

${ }^{17}$ MOLECULE-SWEDEN is an electronic structure program package, written by J. Almlöf, M. R. A. Blomberg, L. G. M. Petterson, B. O. Roos, and P. E. M. Siegbahn. Program modifications to include no-pair relativistic integrals by B. A. Heß, C. M. Marian, and U. Wahlgren.
${ }^{18}$ MOLCAS version 3, K. Andersson, M. R. A. Blomberg, et al., University of Lund, Sweden, 1994. Relativistic no-pair integrals were added by U. Wahlgren.

${ }^{19}$ ECPAIMP is an integral program for ECP and AIMP calculations written by L. G. M. Pettersson, L. Seijo, and M. A. Nygren.

${ }^{20} \mathrm{~K}$. Faegri (private communication with J. Laerdahl).

${ }^{21}$ K. Fægri and J. Almlöf, J. Comput. Chem. 7, 396 (1986).

${ }^{22}$ P. J. Hay, J. Chem. Phys. 66, 4377 (1977).

${ }^{23}$ S. P. Walch, C. W. Bauschlicher, Jr., and C. J. Nelin, J. Chem. Phys. 79, 3600 (1983).

${ }^{24}$ See AIP Document No. E-PAPS: E-JCPSA6-110-307908 for MP parameters and valence basis sets. E-PAPS document files may be retrieved free of charge from our FTP server (http://www.aip.org/epaps/epaps.html) or from ftp.aip.org in the directory /epaps/. For further information, e-mail: paps@aip.org or fax: 516-576-2223.

${ }^{25}$ M. Casarrubios and L. Seijo, J. Chem. Phys. 110, 784 (1999).

${ }^{26}$ T. H. Dunning, Jr., J. Chem. Phys. 90, 1007 (1989).

${ }^{27}$ R. C. Raffenetti, J. Chem. Phys. 58, 4452 (1973).

${ }^{28}$ J. Andzelm, M. Klobukowski, E. Radzio-Andzelm et al., Gaussian Basis Sets for Molecular Calculations, edited by S. Huzinaga (Elsevier, Amsterdam, 1984).

${ }^{29}$ C. W. Bauschlicher, Jr., S. R. Langhoff, H. Partridge, and L. A. Barnes, J. Chem. Phys. 91, 2399 (1989).

${ }^{30}$ A. J. Merer, Annu. Rev. Phys. Chem. 49, 407 (1989).

${ }^{31}$ J. M. Brom, C. H. Durhamm, and W. Weltner, J. Chem. Phys. 61, 970 (1974).

${ }^{32}$ F. Rakowitz, C. M. Marian, and L. Seijo, in preparation. 